\title{
Evaluation of Multicast and Unicast Routing Protocols Performance for Group Communication with QoS Constraints in 802.11 Mobile Ad-hoc Networks
}

\author{
Sumon Kumar Debnath ${ }^{1}$ \\ ${ }^{1}$ Begum Rokeya University, Department of Electrical and Electronic Eng., Rangpur-5400, Bangladesh \\ E-mail: sumon00_ice@yahoo.com
}

\author{
Mousumi Saha ${ }^{2}$, Md. Manowarul Islam ${ }^{3}$, Prodip Kumar Sarker ${ }^{4}$, and Ileas Pramanik ${ }^{4}$ \\ ${ }^{2}$ Okayama University, Dept. of Electrical and Communication Engineering, Okayama, Japan \\ ${ }^{3}$ Jagannath University, Department of Computer Science and Engineering, Dhaka-5400, Bangladesh \\ ${ }^{4}$ Begum Rokeya University, Department of Computer Science and Engineering, Rangpur-5400, Bangladesh \\ E-mail: \{mousumisahamou0, mano.cse.du, sarker.bsmrstu, ileass\}@gmail.com
}

Received: 13 June 2020; Accepted: 09 September 2020; Published: 08 February 2021

\begin{abstract}
In Mobile Ad-hoc Network (MANET), portable devices like smartphones, or laptop PC can join together to make provisional networks without any infrastructure The objective of multicast or unicast protocols is to ensure an efficient route formation and flow control mechanism which is a very challenging issue for many group computing services in MANETs. MANETs can support several real-time applications like emergency rescue, and disaster relief operations which require minimum Quality of Service (QoS) to handle high traffic. Providing QoS for multimedia and group-oriented computing in MANETs becomes a real challenge due to the wireless medium and the mobility of operating nodes. Therefore, an investigation of routing protocols for one-to-many or many-to-many computing is important that supports acceptable QoS in MANETs. Numerous QoS metrics have been considered for the assessment like packet delivery ratio, latency, packet loss rate, control overhead, and throughput. By considering different network topologies and scenarios with different performance parameters, the primary goal of this study is to explore the challenges and factors for QoS services in MANET's multicast communication. The outcomes of investigation can be used to design the future MANET protocol for multimedia applications. The performance results indicate that the increasing number of sending/receiving nodes may increase the overhead or latency of the network but capable of providing higher network throughput, carried out in NS-2. The results also indicate although MANETs can induce errors and packets are lost as part of the normal operating context, multicast AODV practice superior to the unicast protocol to various QoS in a wide range of scenarios with less overhead.
\end{abstract}

Index Terms: MANETs, Unicast, DSR, Multicast, MAODV, QoS, NS-2.

\section{Introduction}

With the appearance of novel technologies and convenient mobile computing devices, the application of mobile ad-hoc networks (MANETs) has upraised for group computing services. It allows a set of wireless clients to form temporary work-groups and communicate with each other without using an access point (AP) [1,2,3]. MANETs are very interesting in multimedia and group-oriented computing for their quick formation ability of temporary networks. Some of these applications are sensible to packet delay but can tolerate the loss of packet and some other operations are packet loss responsive but can accept delay desired for real-time applications. Multicasting is an effective method to implement point-to-multipoint and multipoint-to-multipoint services but it needs networks with QoS enabled multicast routing which is very complex issues in MANETs [4,5,6]. Because the individual applications may have specific QoS constraints and therefore the QoS models are more important in MANETs. Therefore, several QoS facilities must be unified into MANETs to make it suitable for multicast communication. A certain form of admission-control and resource-reservation concept are requested in MANET to deliver various QoS agreements like minimum required packet delivery ratio, throughput and network bandwidth, maximum acceptable packet drop rate, delay, jitter and control overhead $[7,8]$. 
However, it is a challenging issue for MANET nodes to afford reliable group computing facilities and quality of service (QoS) promise. The key factors for this are the unreliable and inconsistent natures of the wireless link, insufficient channel bandwidth, frequent movement of operating nodes, low battery capacity [9]. Multicasting performs an important role in MANETs to concurrently delivering a single message from a data source to a group of receiving nodes [10,11]. Unlike multiple unicast transmission, it can use network resources and bandwidth more efficiently for one-to-many and many-to-many computing. Also, it can reduce the communication overhead, sender/router processing, and delay of the network with identical reliability as multiple unicast concepts $[12,13]$. Figure 1 shows the benefits of multicast service for one-to-many communication. Depending on the application, multicast routing can provide high throughput and low latency for applications like online rescue or video conference. For example in Figure 1 a) the source node has to send duplicate data packets to three destination nodes at the receiving end $(S \rightarrow A \rightarrow B \rightarrow C \rightarrow D 1 ; S \rightarrow$ $\mathrm{A} \rightarrow \mathrm{B} \rightarrow \mathrm{E} \rightarrow \mathrm{D} 2$ and $\mathrm{S} \rightarrow \mathrm{A} \rightarrow \mathrm{B} \rightarrow \mathrm{F} \rightarrow \mathrm{D} 3$ ). On the other hand, for multicast routing, with IP multicast, a data source creates only a single packet to be delivered to a multicast address and the route node then intelligently forwards that packet to a group of the destination nodes. As a result, multicast routing can reduce the network load and improve the throughputs. Thus, it provides efficient use of limited resources with high QoS performance compared to the unicast routing [14].

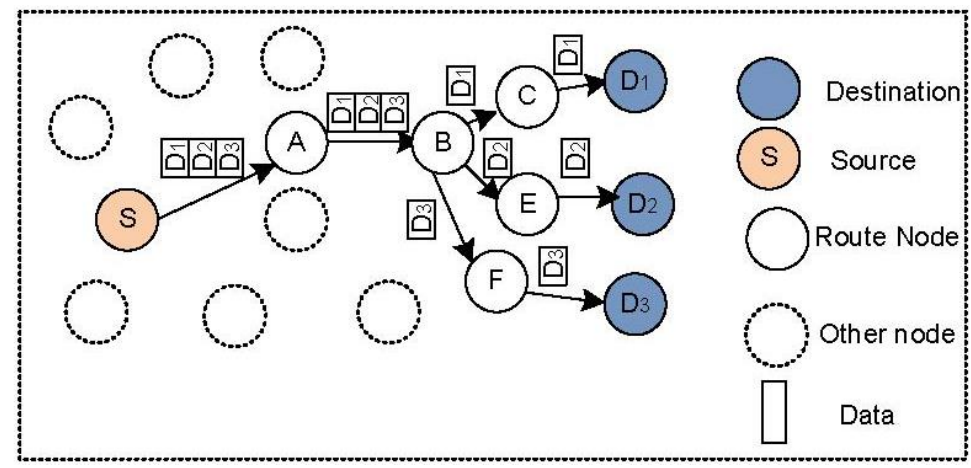

(a) Multiple unicast

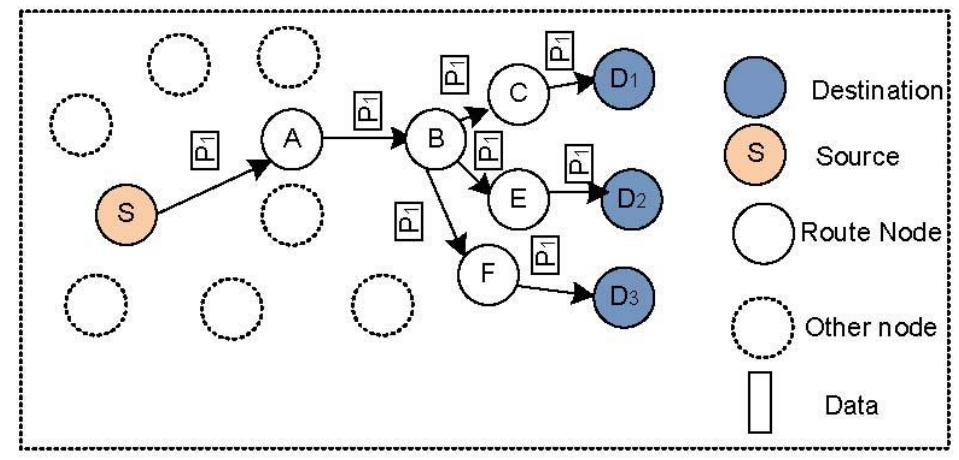

(b) Multicast

Fig.1. Influence of multicasting for one-to-many computing

In literature, a number of review papers have been found those either separately or jointly consider multicast routing. We found that QoS multicast communications in MANETs are reviewed in $[15,16,17,18]$. In [15], the authors surveyed challenges in providing QoS aware multicast communication in MANET for real-time and non-real-time scenarios. In [16], the authors investigated seven QoS multicast routing protocols focusing on their challenges and solutions. In [17], ten QoS multicast routing protocols were examined. All of the above three papers have included only a portion of QoS provisioning multicast protocols in MANETs. In [18], the authors provided a detailed survey of thirtyone QoS enable multicast routing protocols for MANETs. But, the perceptions of multicasting and QoS were not much highlighted.

To provide a better understanding we investigate various factors that affect the performance of to find the challenges and factors of MANETs routing protocols for QoS aware multicast communication. Besides, we evaluate the performance of multicast communication in MANETs for group-oriented computing services by considering different network topologies and scenarios with numerous QoS metrics. The multicast ad-hoc on- demand distance vector routing protocol (MAODV) has been used for performance evaluation. For performance comparison unicast Dynamic Source Routing (DSR) protocol $[19,20]$ is being considered. In summary:

- Firstly, various factors those are essential to provide high-quality performance for different applications like emergency rescue, and disaster relief operations, video streaming is addressed. 
- Secondly, various QoS metrics such as the packet delivery ratio (PDR), data throughput, average latency, normalized routing overhead (NRO), normalized MAC load (NML), network control overhead (NCO), and packet loss rate (PLR) are considered while the total amount of 1) sending nodes and 2) receiving nodes, and 3) the data sending rate of the source nodes are varied.

- Finally, to show the effectiveness of the multicast routing with different network scenarios, multicast AODV protocol has been considered for performance comparison with unicast DSR protocol.

The rest sections of the paper are prepared as follows: In section 2, we briefly mention some related research works. In section 3, a review of QoS and multicast routing concept for MANETs is presented. In section 4, we present the simulation environment and QoS metrics used for performance investigation. In section 5 and section 6, we discuss the simulation results and provide comments based on the investigation. Finally, we conclude the paper in section 7.

\section{Related Works}

Various related researches have been perceived in the literature to investigate the QoS performance of MANETs protocols.

Perkins et al., in [21] implemented a unicast AODV routing protocol in MANETs and compared them with DSR in terms of four QoS constraints (packet delivery ratio (PDR), normalized routing load (NRL), normalized MAC load (NML), and packet delay) through NS-2 simulation. The performance difference is executed for various nodes movement, traffic load, and size of the network. In [22], authors evaluated a comparative study of unicast proactiveOLSR, reactive-AODV, and hybrid-DZTR MANETs routing protocols in connection with the PDR, NRL, and packet delay by changing only the total mobile nodes in the topology. In [23], the authors examined two reactive unicast AODV and unicast DSR protocols using NS-2 concerning PDR, NRL, NML, and delay by changing the amount of data sources, the speed of nodes and their pause time.

In [24], the authors investigated and compared two reactive multicast AODV (MAODV) and ODMRP protocols in the matter of PDR and latency under various traffic load, node speed, the area of simulation. Their simulation result shows that multicast AODV works well with huge data traffic and ODMRP functions well for large network topology and high node mobility. In [25] the authors propose a power based method for improving the performance of Multicast ODMRP protocol. In [26] the author evaluates the performance of multicast AODV and unicast AODV protocols in VANET models by comparing two QoS parameters ( PDR and end-end-delay). In [27] authors provide a comparative study between unicast DSR and multicast ADMR routing protocols in VANETs with various data rates of nodes and three QoS metrics (PDR, NRO, and packet delay).

Sumon et al. in [9] compare the various QoS performance of unicast and broadcast routing protocols in MANETs by varying the number of senders/receivers.

From the above discussion, it is clear that most of the existing works do not consider all the metrics related to the high-level quality of services for MANETs. Either they consider the unicast protocols for point-to-point communication or use the least number of parameters. To deal with various network design scenarios in this paper, we investigate the most interesting metrics parameter to evaluate a multicast routing protocol.

\section{Qos and Multicast Routing over Manets}

In this section, we briefly review the multicasting concept and explore the challenges and factors for quality of service (QoS) multicast routing in MANET.

Multicasting can improve the efficiency of the wireless network by sending a unique copy of a packet to multiple receivers. Thus, reliable multicast data delivery can play significant roles in MANETs especially for various application areas like an online classroom, video conferencing, video-on-demand services and disaster management, etc. However, effective and reliable multicast packet transmission is very difficult and challenging.

Maintaining an adequate level of QoS is one of the greatest challenges in protocol designing for MANET multicasts. To obtain QoS requirements such as capacity, delay, delay jitter, and packet loss the multicast routing protocols should be able to proper use of different network resources. Due to the limited transmission range, bandwidth, and node mobility, it is very difficult to meet all QoS requirements concurrently.

Besides, the large number of receiver nodes may make the packet transmission process complex. Thus, it is better to investigate and evaluate the protocol under different conditions of the network and also find the level of QoS by varying the number of sender and receiver nodes. To observe the effect of the different network settings, we have to evaluate the multicast AODV considering various parameters related to QoS. For the performance comparison, we have considered the unicast DSR protocol. What follows we describe the basic operations of multicast AODV routing protocols with appropriate example. 


\subsection{Multicast AODV (Ad-hoc On-demand Distance Vector Routing) Protocols}

Multicast AODV (MAODV) [28] is a modified form of AODV protocol that can offer reliable communication among groups of people in MANETs. It is the multicast reactive protocol where the source node discovers a bidirectional joint multicast tree to form one or more groups. The tree made of connective and group member nodes created an on-demand basis. The first node of the group acts as a group leader responsible to manage all group members by using a unique address and group sequence number. Though it offers loop-free routing, fast adaptation to the environment, low routing or memory overhead, and low deployment of network it has less connectivity than meshbased routing.

In multicast AODV, each mobile node keeps three routing tables to afford the multicast operation. The first one is known as unicast-route-table used to register the unicast address of the next-hop for a unicast path to other nodes in the network. The second is the multicast-route-table that contains the next-hop address for the tree structure of every multicast group. Finally, the third one is the group-leader-table that records the existing multicast-group with its group leader address and the address of the next-hop to that group leader. MAODV routing mechanism has two main phases: 1) route discovery and 2) route maintenance phase.

During the route discovery phase, a node initiates route-request (RREQ) or RREQ-J (set join flag) message when it wants to obtain a complete path toward a multicast-group for sending packets or simply wish join to a group. This RREQ is usually unicast or broadcast subject to the current info that exists at the source node's routing tables and if needed anyone group member of the multicast tree can answer to the RREQ. Upon getting RREQ a node updates the next-hop info and sequence number for the data source into its routing tables. This inverse path info is used to guide the route-reply (RREP) message to the data source. A node of the preferred multicast tree only can reply to an RREQ-J. If it is not an RREQ-J, then a node with a new path to the multicast-group can reply directly (whose group sequence number > RREQs). It is also updating its unicast-route-table and multicast-route-table using the info about the next-hop to the path of requesting node and unicasts the RREP back to that node. Every node along the path adds the entry in the unicast-route-table for the node from which they received the RREP creating the forward path. When a non-target group node receives RREQ, it just sends that message to its neighbor nodes. Figure 2 (a) shows an example of RREQ/RREP message propagation in MAODV and Figure 2(b) does the joining operation of a potential node.

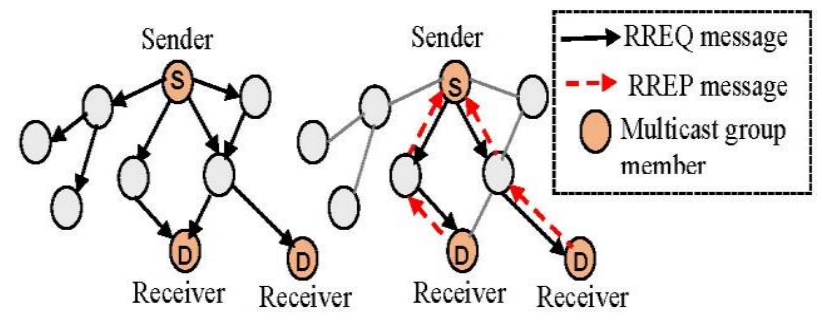

2. RREQ RREP

(a) The route discovery process in MAODV

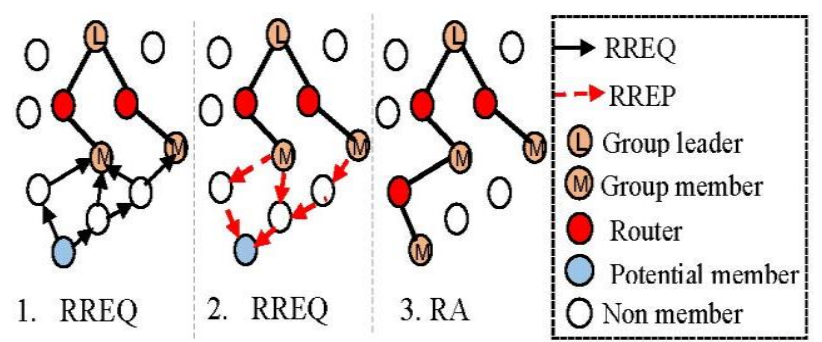

(b) Joining operation of a potential node to a multicast group.

Fig.2. MAODV operation

Due to the movement of operating nodes in MAODV, one or more links on the shared tree may be destroyed. Besides, a node can wish to withdraw its membership state from the group anytime or there may be needed to rejoin partitioned trees. In all the above situations, MAODV performs tree link or route maintenance procedure which is invoked in an appropriate time to exploit the connectivity of multicast-group.

\section{Simulation Tools}

To investigate the performance of unicast DSR and multicast AODV (MAODV) MANETs routing protocols for one-to-many and many-to-many traffic, simulation is conducted using NS-2.34. Because NS-2 is an open-source 
simulator commonly used by the research academia for the evaluation of any proposed protocols/algorithm in 802.11 ad-hoc networks [29,30]. Moreover, it offers a convenient environment for the modeling, simulating TCP/UDP, and various unicast, multicast, and IP protocols over conventional networks. The NS-2 package consists of 1) toolcommand language (TCL) for open-source scripting, 2) an extension of TCL (OTCL) for object-oriented scripting, 3) TCLCL: TCL/C++ interface, and network simulator version 2.

All simulations are done in NS-2.34 by changing the number of sending/receiving mobile nodes and their data sending rate where a total of 50 mobile nodes are located in the network field of size $1500 \mathrm{~m} \times 300 \mathrm{~m}$. By OTCL scripting language in NS-2, we set up the network topologies, and create a radio propagation model, and the movement scenario arrangement of nodes. We also create the pattern of the traffic sources and other necessary applications. We use the shadowing model [9] as a radio propagation model that attempts to model a more realistic simulation environment. For the traffic and mobility model, we use the constant bit rate (CBR) traffic and random waypoint mobility (RWP) model [9].

The outcome of the simulation is recorded in the trace files which are analyzed later by using the trace graph or Perl scripting language to evaluate various QoS metrics. We also visualize the whole simulation processes using the network animator (NAM). Table 1 shows PC configuration used for simulation and table 2 does the simulation parameters that we configured in NS-2.

Table 1. PC configuration for simulation.

\begin{tabular}{|c|c|}
\hline model & Ultrabook lesance NB S344/L \\
\hline CPU & Intel(R) core i5-3317U $(1.7 \mathrm{GHz})$ \\
\hline wireless adapter & Qualcomm Atheros AR9285 \\
\hline RAM & 4 GB \\
\hline OS & Linux Redhat 9.0 \\
\hline
\end{tabular}

Table 2. Simulation parameters in NS-2

\begin{tabular}{|c|c|}
\hline Parameter & Value \\
\hline Channel & Wireless \\
\hline PHY/Mac layer & DCF of IEEE 802.11 WLAN \\
\hline Carrier sense & CSMA/CA \\
\hline Radio interface model & 802.11 DSSS Lucent WaveLAN \\
\hline Channel capacity & $2 \mathrm{Mbit} / \mathrm{sec}$ \\
\hline Transmission range & $250 \mathrm{~m}$ \\
\hline Carrier sensing range & $471.5 \mathrm{~m}$ \\
\hline Interface queue (IQ) & CMUPriQueue \\
\hline Interface queue length & 50 packets \\
\hline Antenna type & OmniAntenna \\
\hline Propagation model & Shadowing path loss model \\
\hline Frequency & $9.14 \times 10^{8} \mathrm{~Hz}$ \\
\hline Path loss exponent & 2.0 \\
\hline Standard deviation & 4.0 \\
\hline CPThreshold & 10 Watt \\
\hline RXThreshold & $6.76 \times 10^{-10}$ \\
\hline CSThrehold & $2.89 \times 10^{-11}$ \\
\hline Power $(\mathrm{Pt})$ & $0.28 \mathrm{Watt}$ \\
\hline System Loss & 1.0 \\
\hline Traffic model & CBR traffic \\
\hline Packet size & 512 bytes/packet \\
\hline Mobility model & Random waypoint model \\
\hline Pause Time & $0 \mathrm{~m} / \mathrm{s}$ \\
\hline Max. Speed & $20 \mathrm{~m} / \mathrm{s}$ \\
\hline CBR rate & $2 \mathrm{pkts} / \mathrm{sec}$ \\
\hline Each packet size & 512 bytes \\
\hline Area of simulation & $1500 \mathrm{~m} \times 300 \mathrm{~m}$ \\
\hline Routing protocols & Unicast DSR, multicast AODV \\
\hline Simulation time & $200 \mathrm{~s}$ \\
\hline
\end{tabular}




\subsection{QoS factors for performance evaluation}

The performance of unicast DSR and multicast AODV (MAODV) protocol are investigated by considering the following seven QoS performance metrics [23, 31, 32]:

- Packet delivery ratio (PDR): It states the relationship between the total packets received successfully at the final endpoint by the CBR sink and total packets transmitted by the CBR source.

$$
\operatorname{PDR}(\%)=\frac{\sum_{1}^{N} C B R_{\text {recv }}}{\sum_{1}^{N} C B R_{\text {Send }}} \times 100
$$

- Average latency: In MANET, packets are provisionally buffered through the discovery of route, delay at the IQ, and in MAC layer packet retransmission, propagation, and transfer time. The average latency contains all of the above delays qualified by each packet traveling from source to destination. Low latency means better performance of applications and it is defined as

$$
\text { Latency }=\frac{\sum_{1}^{n}\left(C B R_{\text {sendTime }}-C B R_{\text {recrTime }}\right)}{\sum_{1}^{n} C B R_{\text {recv }}}
$$

- Packet loss rate (PLR): It expresses the portion of packets dropped either at the source/intermediate nodes in the network.

$$
P L R=\frac{\sum_{1}^{n}\left(C B R_{\text {recev }}-C B R_{\text {send }}\right)}{\sum_{1}^{n} C B R_{\text {Send }}}
$$

- Normalized routing overhead (NRO): It examines the routing protocols efficiency and is defined as:

$$
N R O=\frac{\text { Number of routing packets transmitted }}{\text { Number of data packets received }}
$$

- Normalized MAC load (NML): This metric calculates the actual exploitation of the wireless medium by data traffic. It is defined as the amount of routing, Address Resolution Protocol (ARP), and RTS, CTS, and ACK control packets delivered by the MAC layer for every transmitted data packet.

- Network control overhead (NCO): NCO includes both the overhead during routing packets and the MAC overhead and is defined as:

$$
N C O=\frac{\text { Number of transmitted control packets }}{\text { Number of received data packets }}
$$

- Throughput: It states the ratio of the total packets received through the receiving node and the time difference of the last and first received packet.

$$
\text { Throughpt }=\frac{\text { rxBytes } * 8}{\text { TimeLastRxpkt-TimeFirstRxpkt }}
$$

Where rxBytes is the total amount of received packets. TimeLastRxPacket is the absolute time of the last received packet and TimeFirstRxPacket does the total time of the first packet. 


\section{Simulation Results for Various Number of Sending/Receiving Nodes}

In this portion, we evaluate various QoS matrices of unicast DSR and multicast AODV (MAODV) protocols to show the effect of the total data sending or receiving nodes for multipoint data communication in MANETs.

\subsection{QoS results for various number of sending nodes for group communication}

To evaluate the impact of the total quantity of traffic sources on various QoS factors for group computing in MANETs, this simulation is performed using identical traffic and network scenarios for both unicast DSR and multicast AODV protocols when we rise the total amount of data sending nodes from 1 node to 10 nodes $(1,2,5,7$, and 10 source nodes) while keeping the total number receiving nodes constant to 20 nodes. We run each simulation instance 10 times with a duration of 200 s and finally we take the average results. The various QoS performance results such as the PDR, throughput, avg. latency, NRO, NML, NCO, and PLR are plotted in Figure 3. From these graphs in Figure 3, it is perceived that through increasing the total number of data sending nodes, the PDR and throughput of multicast AODV are higher than the unicast DSR. On the other hand, the avg. latency, NRO, NML, NCO, and PLR of multicast AODV is much lower than the unicast DSR protocol as in figure 3. An acceptable QoS is the key measurement of the overall performance of MANETs in many applications which includes data sharing among groups of people or multipoint conferences. In this case, unicast DSR gives poor QoS than multicast AODV.

(a) Number sending nodes vs. PDR
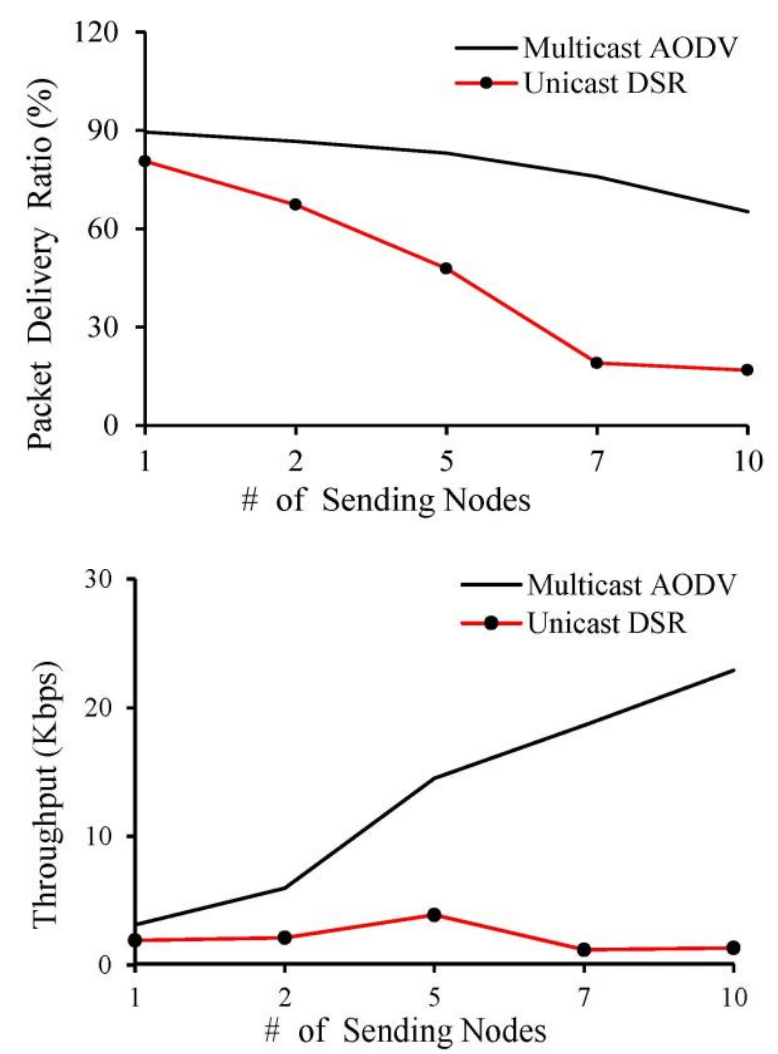

(b) Number sending nodes vs. data throughput

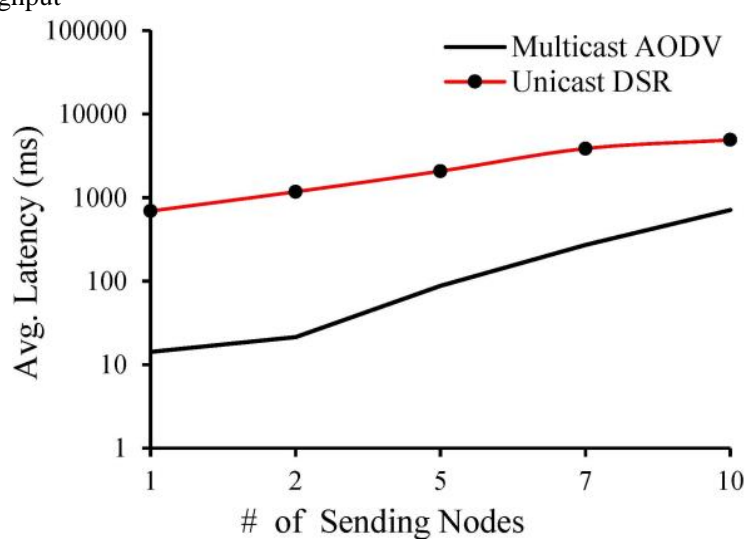

(c) Number sending nodes vs. packet latency 
(d) Number sending nodes vs. NRO
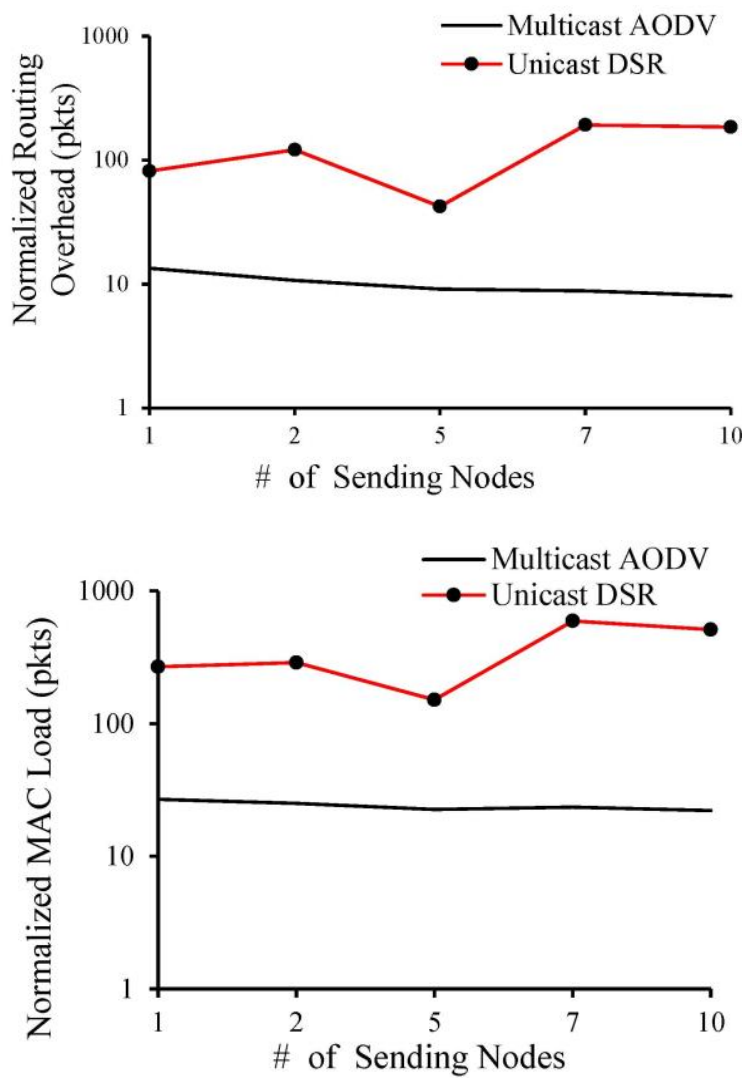

(e) Number sending nodes vs. NML

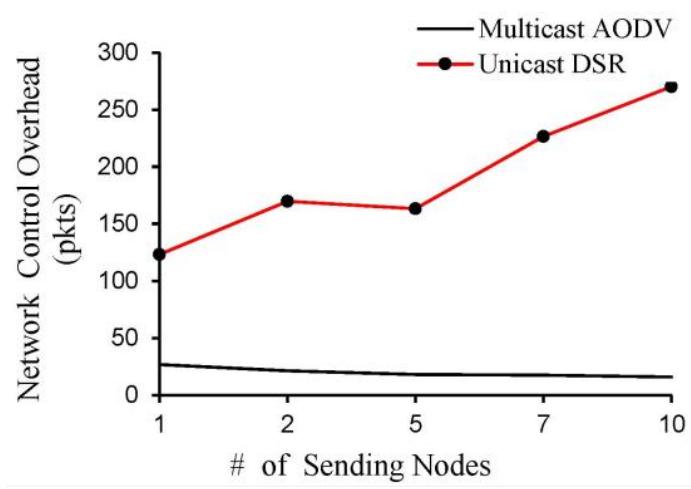

(f) Number sending nodes vs. NCO

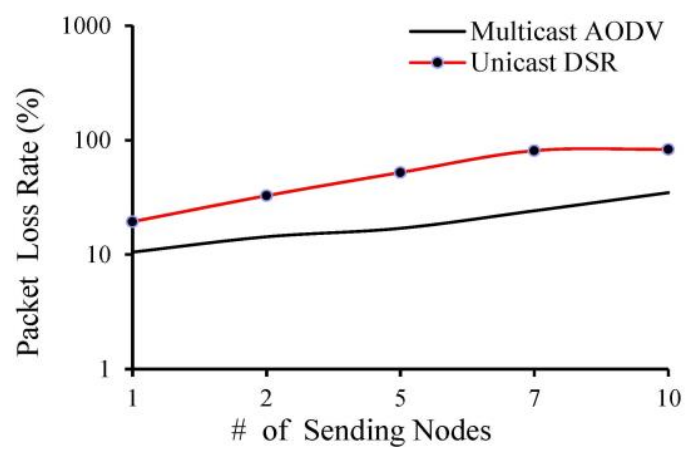

(g) Number sending nodes vs. PLR

Fig.3. QoS results with various number of sending nodes

The QoS results of unicast DSR become worse drastically unlike multicast AODV when the number of data sending nodes becomes two or more. Because in this case, a vast amount of traffics is generated throughout the MANETs since the unicast DSR needs to set up dedicated unicast connections between each of the traffic sending nodes and each of the receiving nodes in the group. For example, consider a network scenario that has $\mathrm{N}$ nodes performing as a traffic source and $\mathrm{M}$ nodes as a traffic receiving node, thus unicast DSR needs to detect and preserve a total $\mathrm{N} \times \mathrm{M}$ simultaneous unicast links to deliver messages to $\mathrm{N}$ nodes. Each source node using unicast DSR protocol 
also needs to generate $M$ replicas of packets to be sent to $M$ receiving nodes. This multiple unicast transmission in unicast DSR causes more wasteful utilization of wireless links and makes network congestions. Also, it introduces substantial overhead (NRO, NML, NCO, and others) in the network and therefore needs more bandwidth resulting reduction of the QoS rapidly with increasing the multicast-group size.

On the other hand, the use of route adaptation and self-pruning, the multicast AODV significantly reduces network load by transmitting fewer packets while comparing to the unicast DSR. Therefore MAODV gives better QoS performance than the unicast DSR in MANETs.

\subsection{TheQoS results for various number of receiving nodes for group computing}

At this point, we examine the impact of the total amount of data receiving nodes on several QoS elements. This simulation is run using identical network scenarios for both unicast DSR and multicast AODV protocols when we rise the total receiving nodes from 10 nodes to 50 nodes $(10,20,30,40$, and 50 receiving nodes) although the total number dada sending nodes is kept constant to 5 nodes. The various QoS simulation results are plotted in Figure 4.

It can be detected from graphs in Figure 4 that by increasing the total number of data receiving nodes or multicastgroup size, the PDR and the throughput of multicast AODV are also much larger than the unicast DSR. At the same time, the average latency, NRO, NML, NCO, and the PLR of multicast AODV are much lesser than the unicast DSR protocol. The performance of MANETs protocols in group computing such the data sharing among groups of people or multipoint conferences is greatly affected by the satisfactory value of QoS. In this scenario, the unicast DSR also provides poor QoS than the multicast AODV. Because in multicast AODV, the renumber of required transmitted packets is smaller and it consumes least bandwidth and cost than the unicast DSR protocol.

(a) Number of receiving nodes vs. PDR
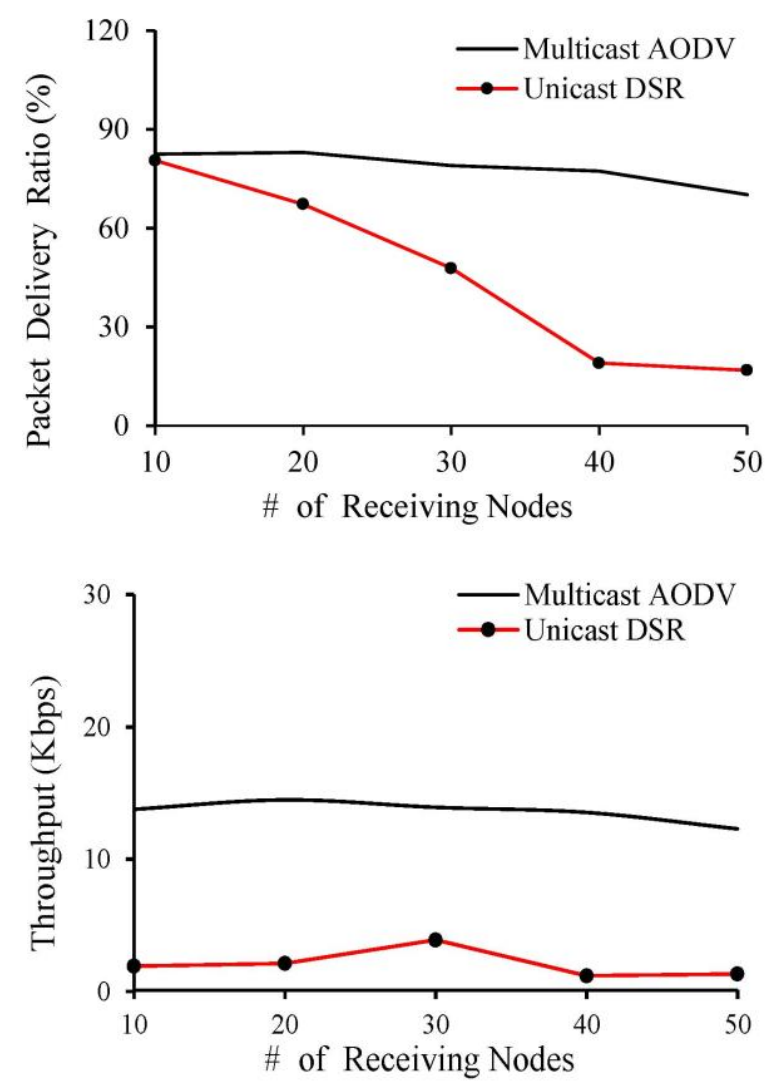

(b) Number of receiving nodes vs. data throughput 
(c) Number of receiving nodes vs. packet latency
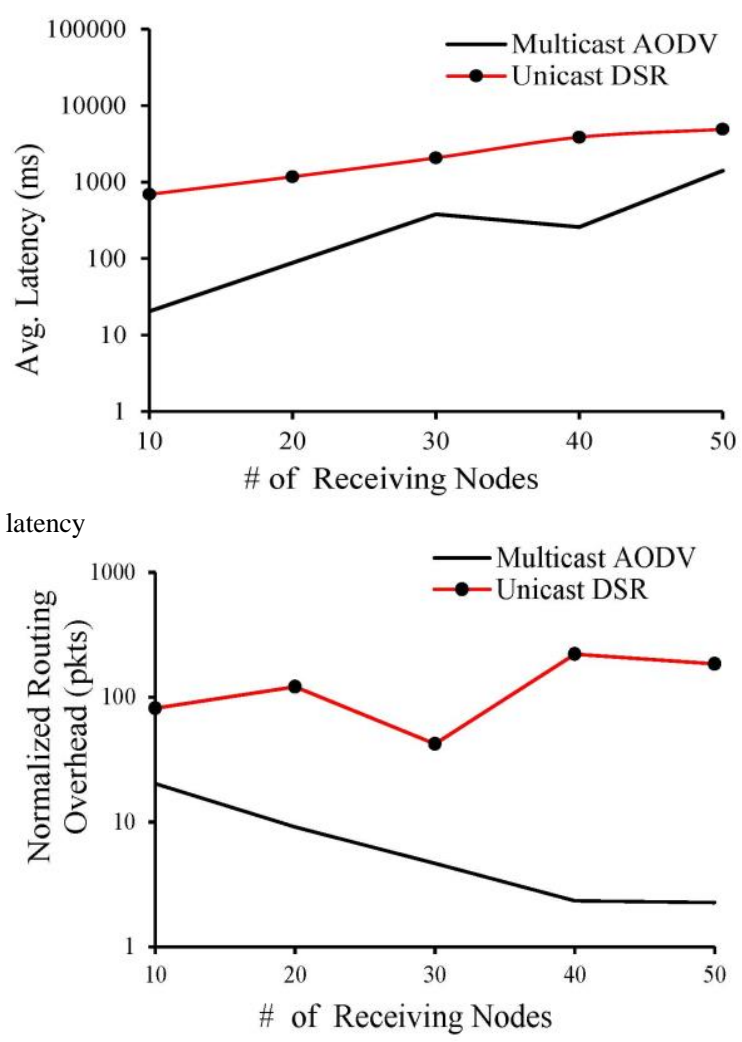

(d) Number of receiving nodes vs. NRO

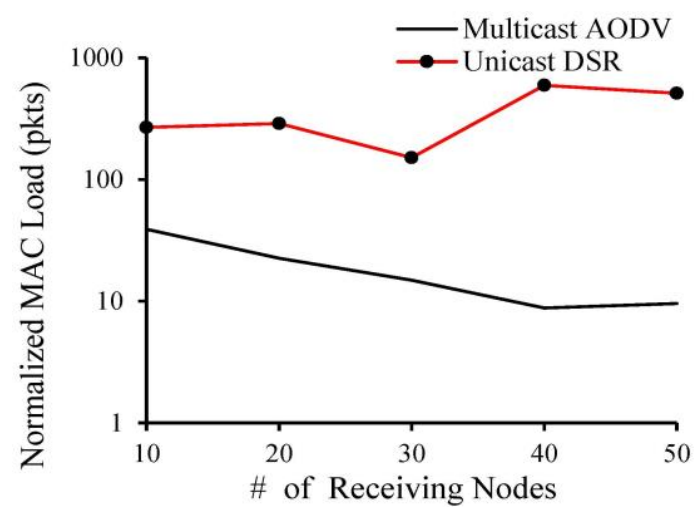

(e) Number of receiving nodes vs. NML

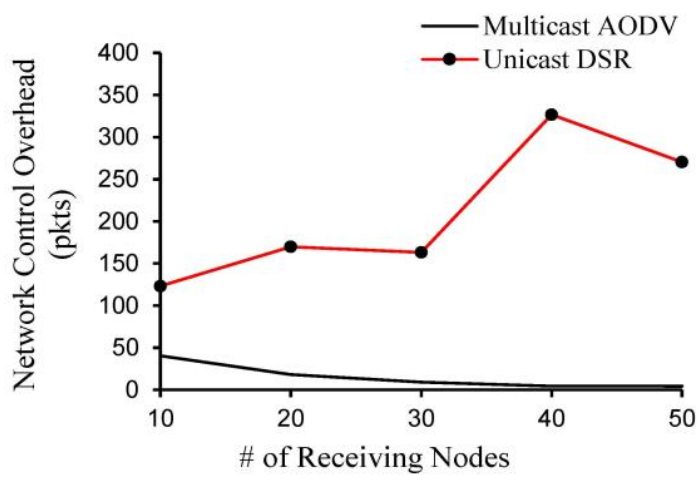

(f) Number of receiving nodes vs. NCO 
(g) Number of receiving nodes vs. PLR

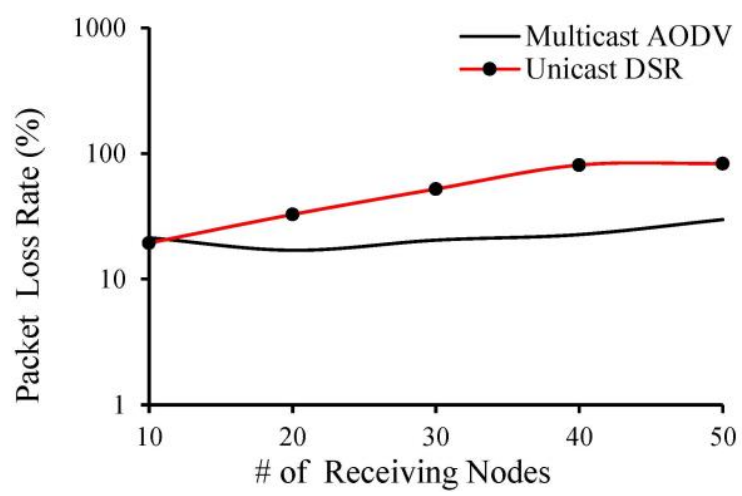

Fig.4. QoS results with various number of receiving nodes

From Figure 4, It is also observed that for 5 data senders and 30 data receivers, the PDR, throughput, latency, NRO, NML, NCO, PLR of multicast AODV and unicast DSR are 79.06\%, $13.92 \mathrm{kbps}, 378.31 \mathrm{~ms}, 4.65$ pkts, 14.91 pkts, 9.201 pkts, $20.44 \%$ and $47.82 \%, 3.9 \mathrm{kbps}, 2076.1 \mathrm{~ms}, 42.23$ pkts, 151.16 pkts, $163.23 \%, 52.18 \%$ respectively.

\section{Simulation Results for Various Number of Data Rates of Source Nodes}

To examine the influence of data rates of source nodes on QoS in MANETs, the simulation is executed for unicast DSR, and multicast AODV routing protocol where the total amount of source and destination nodes is to keep constant at 1 node, and 30 nodes correspondingly. The data rate of each source node is increased from 2 to 20 pkts/sec (2, 4, 8, 12,16 , and $20 \mathrm{pkts} / \mathrm{sec}$ ) and the seven QoS metrics are estimated.

The simulation results are plotted in Figure 5. It can be observed from Figure 5(a) that with rising the data rate, the PDR of unicast DSR, and multicast AODV protocols decreases. The obvious reason behind this is that as the data rates of each source node are increased, the extra packets are injected into the system before dealing with the preceding data. Therefore the queue is the overthrow and sudden packets drop occurred. Figure 5(a) also shows that with various data send rates the PDR of DSR and multicast AODV (MAODV) vary between the range of 82-19\% and 84-49\% respectively and MAODV protocol shows better PDR output. From Figure 5(b), 4(c) and 4(g) it can be perceived that with growing the data rate of source, the throughput, average packet latency, and PLR of DSR, and multicast AODV protocols are varied correspondingly as the total amount of data sending/receiving nodes. Multicast AODV also gives better results than DSR protocols. Similarly, the NRO, NML, and NCO results with numerous data sending rate are revealed in Figure 5(d), 4(e), and 4(f). From Figure 5(d) 4(e), and 4(f) it can be observed that the NRO, NML, and NCO for DSR is high as compared to the MAODV. This is since that with growing the data rate, DSR requires to generate more control packets (routing and MAC packets, address resolution protocol, RTS, CTS, and ACK) than MAODV protocol.

(a) Data sending rate vs. PDR

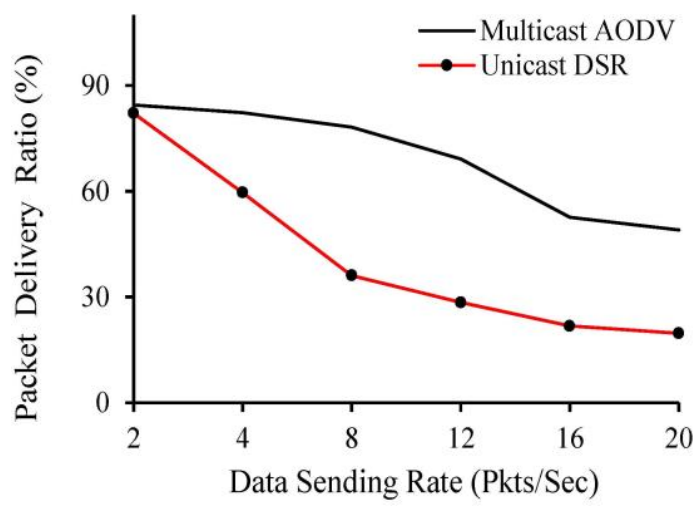


(b) Data sending rate vs. data throughput
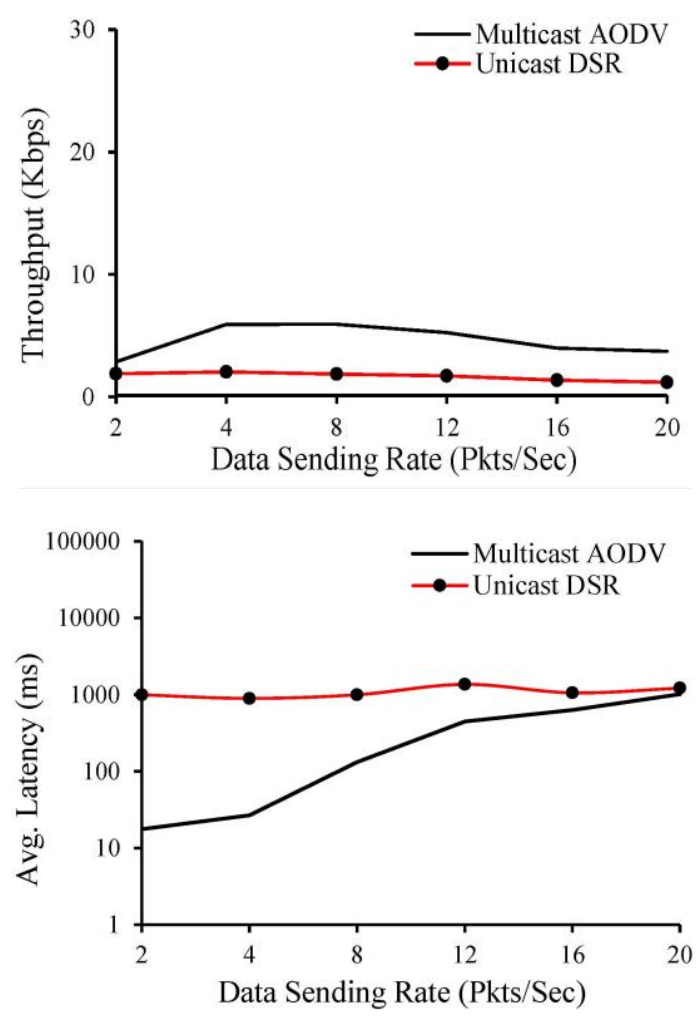

(c) Data sending rate vs. packet latency

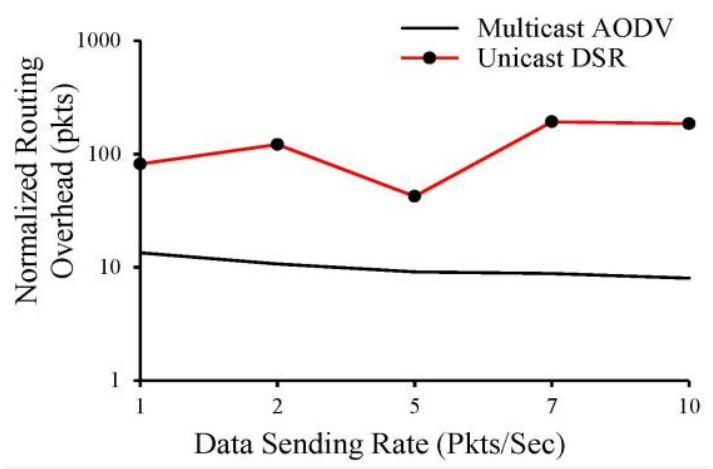

(d) Data sending rate vs. NRO

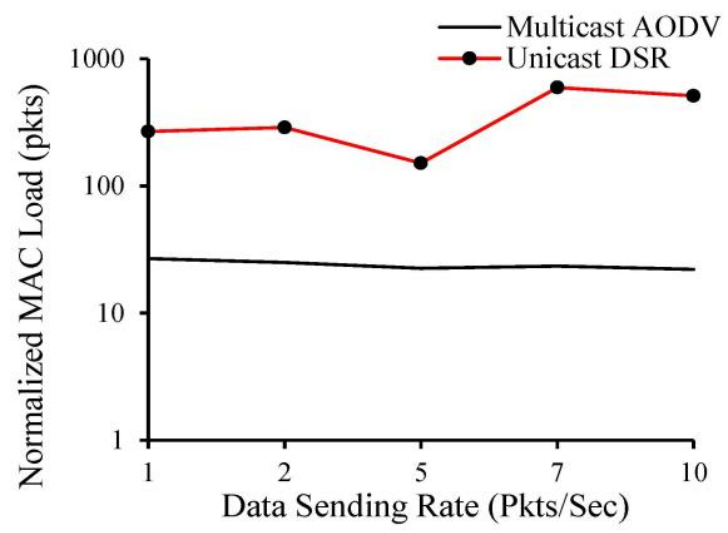

(e) Data sending rate vs. NML 


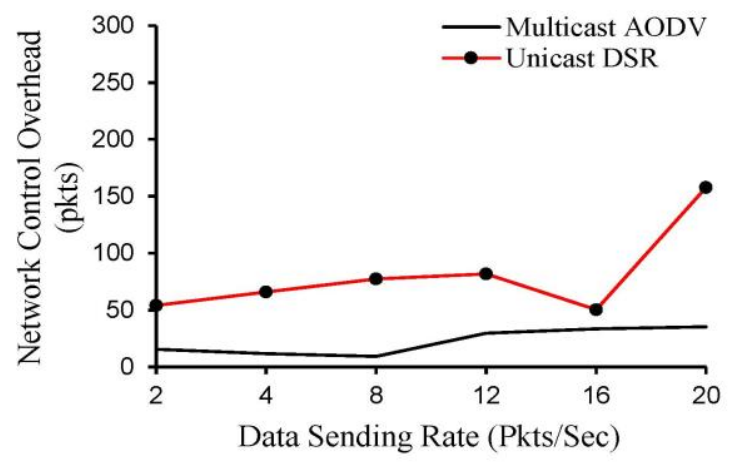

(f) Data sending rate vs. NCO

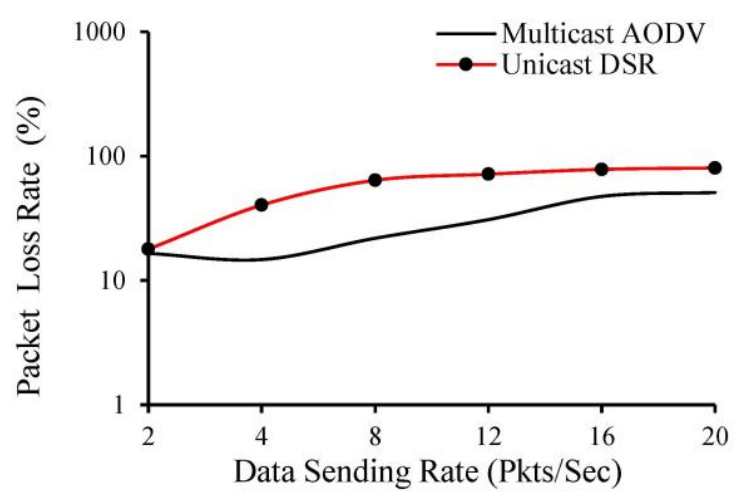

(g) Data sending rate vs. PLR

Fig.5. QoS results with various data rates of source nodes

\section{Conclusions}

This paper investigated the QoS performance of multicast routing protocol for MANETs. Multicast affords enhanced QoS performance than unicast protocol. With the increasing number of sender/receiver nodes and higher data rate, the performance dropped rapidly as the network needs to determine and preserve a lot of unicast links which leads significant network overhead and causes huge utilization of network bandwidth. On the other hand, multicast can utilize the channel bandwidth more efficiently by controlling overhead of the network and it creates less traffic in the network by avoiding the transmission of redundant packets which fastens the system in comparison to the unicast routing protocol. The performance graphs considering many QoS parameters also confirm the effectiveness. Our future works include the more exploration of multicast MANETs routing protocol in diverse network environments and optimal design of the routing protocols in MANETs.

\section{References}

[1] H. Gavin, and N. Vaidya, “Analysis of TCP Performance over Mobile Ad Hoc Networks”, Wireless Networks, Vol. 8, pp. 275288, Mar 2002

[2] S. Salem, and A. El-Bareg, "MANET Testbed using Raspberry PIs", Int. J. of Wireless and Microwave Technologies, Vol. 2, pp. 52-63, 2018. DOI: 10.5815/ijwmt.2018.02.05

[3] T. Alama, and B. Rababahb, "Convergence of MANET in Communication among Smart Devices in IoT", Int. J. of Wireless and Microwave Technologies, Vol. 2, pp. 1-10, 2019. DOI: 10.5815/ijwmt.2019.02.01

[4] E. Chip, and B. Heile, "Self-Organizing, Self-Healing Wireless Networks", IEEE Aerospace conf., vol. 1, pp. 149-156, Mar 2000 .

[5] J. R. McChesney, and R. J. Saulitis, "Optimization of an Adaptive Link Control Protocol for Multimedia Packet Radio Networks", Proc. IEEE Int. Conf. Military Communications, vol. 1, pp. 261-265, Oct 1999.

[6] E. Baburaj, and V. Vasudevan, "An Intelligent Multicast Ad-hoc On demand Distance Vector Protocol for MANETs", J. of Networks, Vol. 3, No. 6, pp. 62-68, June 2008.

[7] S. Biradar, and P. Kulkarni, "Enhancing the Quality of Service using M-AODV Protocol in MANETs", Int. Conf. on Applied and Theoretical Computing and Communication Technology, vol. 9, no. 3, pp. 648-652, Oct 2015.

[8] L. Hanzo and R. Tafazolli, "A Survey of QoS Routing Solutions for Mobile Ad hoc Networks," IEEE Communications Surveys \& Tutorials, Vol. 9, No. 2, pp. 50-70, July, 2007.

[9] S. K. Debnath, F. Ahmed, and N. Islam, "Performance Evaluation of Unicast and Broadcast Mobile Ad-hoc Networks Routing Protocols", Int. J. of Computer Science and Information Security, Vol. 7, No. 1, pp. 040-046, Jan 2010.

[10] B. A. Forouzan, "TCP/IP Protocol Suite", 3rd Edition, 2006. 
[11] S. Baolin, S. Pi, C. Gui, Y. Zeng, B. Yan, W. Wang, and Q. Qin, "Multiple constraints QoS multicast routing optimization algorithm in MANET based on GA", Progress in Natural Science, vol. 18, no. 3, pp. 331-336, Mar 2008.

[12] U. Varshney, "Multicast over Wireless Networks", Communications of the ACM, Vol. 45, No. 12, pp. 31-37, Dec 2002.

[13] J. H. Schiller, "Mobile communications", Pearson education, 2003.

[14] A. Chopra, R. Kumar, "Efficient Resource Management for Multicast Ad Hoc Networks: Survey", Int. J. of Computer Network and Information Security, Vol.8, No.9, pp.48-55, 2016.DOI: 10.5815/ijcnis.2016.09.07

[15] D. Chander, R. Kumar, "A survey of issues in supporting QoS based multicast routing protocols over MANETs", Int. J. of Comp. App., vol. 112, no. 13, Feb 2015.

[16] M. Masoudifar, "A Review and Performance Comparison of QoS Multicast Routing Protocols for MANETs", J. Ad-Hoc Networks, vol. 7, no. 6, pp. 1150-1155, Aug 2009.

[17] A. H. A. Hashim, M. M. Qabajeh, O. Khalifa and L. Qabajeh, "Review of Multicast QoS Routing Protocols for Mobile Ad Hoc Networks", IJCSNS International J. of Comp. Sci. and Netw. Sec., Vol. 8, No. 12, pp. 108-116, Dec 2008.

[18] V. T. M. Do, L. Landmark and O. Kure, "A Survey of QoS Multicast in Ad Hoc Networks", Future Internet, vol. 2, no. 3, pp. 388-416, Sep 2010.

[19] D. B. Johnson, and D. A. Maltz, “The Dynamic Source Routing Protocol for Mobile Ad Hoc Networks (DSR)", IETF MANET working group Internet draft, July 2004.

[20] G. Najafi, S. J. Gudakahriz, "A Stable Routing Protocol based on DSR Protocol for Mobile Ad Hoc Networks", Int. J. of Wireless and Microwave Technologies, Vol.8, No.3, pp. 14-22, 2018. DOI: 10.5815/ijwmt.2018.03.02

[21] C. E. Perkins, E. M. Royer, S. R. Das, and M. K. Marina, "Performance Comparison of Two On-demand Routing Protocols for Ad hoc Networks", IEEE Personal Communications Magazine special issue on Ad hoc Networking, vol. 8, no. 1, pp. 16-28, Feb 2001.

[22] M. Abolhasan, T. A. Wysocki, and J. Lipman, "Performance investigation on three-classes of MANET routing Protocols", Asia-Pacific Conference on Communications, pp. 774-778, Oct 2005.

[23] G. Jayakumar, and G. Ganapathy, "Performance Comparison of Mobile Ad-hoc Network Routing Protocol”, Int. J. of Computer Science and Network Security, VOL.7 No.11, pp. 77-84, Nov 2007.

[24] K. Mishra, and S. Choudhary, "Investigation of Effective Group Communication using MAODV and ODMRP Routing in MANET", Int. J. of Engineering Technology and Applied Science Communications of the ACM, Vol. 2, No. 1, pp. 31-37, Jan 2016.

[25] A..Ghafouri, A. Ghasemi, M. R. H. Ahangar, "A Power-based Method for Improving the ODMRP Protocol Performance in Mobile Ad-hoc Networks", Int. J. of Wireless and Microwave Technologies, Vol.8, No.2, pp. 27-36, 2018. DOI: 10.5815/ijwmt.2018.02.03

[26] M. A. Al-Shabi, "Evaluation the performance of MAODV and AODV protocols in VANETs Models", Int. J. of computer science and information security, vol. 14, no. 1, pp. 1-12, 2020.

[27] P. Shingh, "Comparative study between unicast and multicast routing protocols in different data rates using VANET", Int. Conf. on Issues and Challenges in Intelligent Computing Techniques, pp. 278-284, Feb 2014.

[28] E. M. Royer, and C. E. Perkins, "Multicast operation of the ad-hoc on-demand distance vector routing protocol", In Proceedings of the 5th annual ACM/IEEE Int. Conf. on Mobile computing and networking, pp. 207-218, Aug 1999.

[29] "Network simulator ns-2", https://www.isi.edu/nsnam/ns/ (accessed 12 May 2020).

[30] K. Fall, and K. Varadhan, "The ns Manual (formerly ns Notes and Documentation)", The VINT Project, UC Berkeley, LBL, USC/ISI, and Xerox PARC, Sep 2008.

[31] L. Wang, Y. Shu, M. Dong, L. Zhang, and O. W. W. Yang, "Adaptive Multipath Source Routing in Ad Hoc Networks", IEEE Int. Conf. on Communications, vol. 3, pp. 867-871, Jun 2001.

[32] M. K. Marina, S. R. Das, "Ad hoc on-demand multipath distance vector routing", Wireless communication and mobile computing, vol. 6, no. 7, pp. 969-988, Nov 2006.

\section{Authors' Profiles}

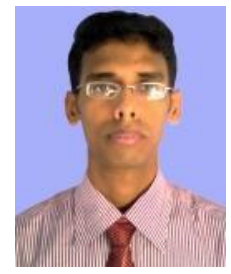

Sumon Kumar Debnath received his B. Sc. And M. Sc. degree in Information and Communication Engineering from Rajshahi University, Bangladesh, in 2008. He received his Ph.D. degree from Okayama University, Japan. He is currently an associate professor at Begum Rokeya University, Bangladesh. His research interest includes wireless networks.

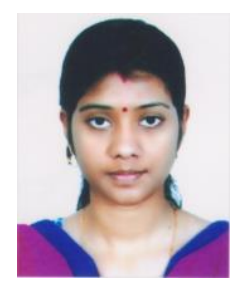

Mousumi Saha received B.Sc. degree in Telecommunication and Electronic Engineering from Hajee Mohammad Danesh Science and Technology University, Bangladesh, in 2013. She also completed her master's degree from Okayama University in 2017. She is currently a Ph.D. student in the Graduate School of Natural Science and Technology at Okayama University, Japan. Her research interest includes wireless communication and networking. 


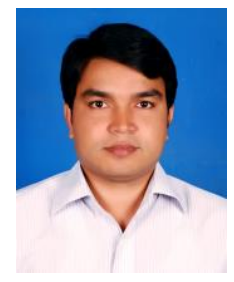

Md. Manowarul Islam received B.S. and M.S. degrees in Computer Science and Engineering from the University of Dhaka, Bangladesh, in 2008 and 2010. He also completed his Ph.D. from the Graduate School of Natural Science and Technology at Okayama University, Japan. He is currently an associate professor at Jagannath University, Dhaka, Bangladesh. His research interests include wireless communication, wireless sensor network, cloud computing.

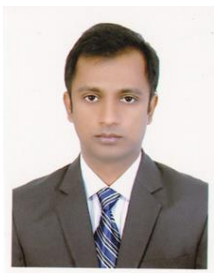

Prodip Kumar Sarker received B.S. and M.S. degrees in Computer Science and Engineering from Rajshahi University, Bangladesh, in 2007 and 2008. He is currently an assistant professor at Begum Rokeya University, Rangpur, Bangladesh. His research interest includes wireless networks, computer vision.

Ileas Pramanik received B. Sc. And M. Sc. degree in Information and Communication Engineering, Bangladesh, in 2008 and 2010. $\mathrm{He}$ also received his Ph.D. degree from City University Hong Kong. His research interests include security and criminal investigation in big data, wireless communication, and wireless sensor network.

How to cite this paper: Sumon Kumar Debnath, Mousumi Saha, Manowarul Islam, Prodip Kumar Sarker, Ileas Pramanik, "Evaluation of Multicast and Unicast Routing Protocols Performance for Group Communication with QoS Constraints in 802.11 Mobile Ad-hoc Networks", International Journal of Computer Network and Information Security(IJCNIS), Vol.13, No.1, pp.1-15, 2021. DOI: $10.5815 / \mathrm{ijcnis.2021.01.01}$ 